Castro-Sánchez, M.; Chacón-Cuberos, R.; González-Valero, G.; Puertas-Molero, P.; Muros, J.J. y Zurita-Ortega, F. (2019) Self-Concept, Physical Activity and Harmful Substances: A Model of Structural Equations. Revista Internacional de Medicina y Ciencias de la Actividad Física y el $\begin{array}{lllll}\text { Deporte } & \text { vol. } & 19 & (75) & \text { pp. }\end{array}$ Http://cdeporte.rediris.es/revista/revista75/artautoconcepto1064.htm DOI: $10.15366 /$ rimcafd2019.75.008

\title{
ORIGINAL
}

\section{AUTOCONCEPTO, ACTIVIDAD FÍSICA Y SUSTANCIAS NOCIVAS: UN MODELO DE ECUACIONES ESTRUCTURALES}

\section{SELF-CONCEPT, PHYSICAL ACTIVITY AND HARMFUL SUBSTANCES: A MODEL OF STRUCTURAL EQUATIONS}

\author{
Castro-Sánchez, M.'; Chacón-Cuberos, R. ${ }^{1}$; González-Valero, G.. ${ }^{2}$ Puertas- \\ Molero, P. ${ }^{2}$; Muros, J.J. ${ }^{1}$ y Zurita-Ortega, F. ${ }^{1}$ \\ ${ }^{1}$ Dr. Educación Física. Universidad de Granada (España) manuelcs@ugr.es, rchacon@ugr.es, \\ iimuros@ugr.es, felixzo@ugr.es \\ 2 Investigador del grupo HUM-238 de la Universidad de Granada (España) \\ gabri1322@correo.ugr.es, pilarpuertas@correo.ugr.es
}

Código UNESCO: 6114.03 Psicología social. Comportamiento Colectivo / Social psychology. Collective Behaviour

Clasificación del Consejo de Europa: 15. Psicología del deporte / Sport Psychology

Recibido 17 de noviembre de 2017 Received November 17, 2017

Aceptado 13 de noviembre de 2018 Accepted November 13, 2018

\section{RESUMEN}

La presente investigación pretende definir y contrastar un modelo explicativo del consumo de alcohol y tabaco, y la práctica de actividad física en función de las cinco dimensiones del autoconcepto en una muestra de 2.134 adolescentes de la provincia de Granada (España). Los instrumentos empleados fueron el Cuestionario de Autoconcepto Forma-5, Test para la Identificación de Trastornos en el Uso de Alcohol-AUDIT, Test para la Dependencia a la Nicotina-FTND y un cuestionario ad-hoc para controlar la práctica de actividad física. En esta investigación se planteó un modelo de ecuaciones estructurales que se ajustó de forma adecuada $(X 2=79,476 ; \mathrm{gl}=8 ; \mathrm{p}=0,000 ; \mathrm{CFI}=0,968 ; \mathrm{NFI}=0,964 ; \mathrm{IFI}$ $=0,968 ;$ RMSEA $=0,065)$. Los resultados obtenidos muestran una relación positiva entre el consumo de alcohol y el consumo de tabaco, y relaciones negativas entre el autoconcepto familiar, emocional y académico con el consumo de alcohol. 
PALABRAS CLAVE: autoconcepto; tabaco; alcohol; actividad física; adolescentes.

\section{ABSTRACT}

The present research aims to define and contrast an explanatory model of alcohol and tobacco consumption, and the practice of physical activity according to the five dimensions of self-concept in a sample of 2.134 adolescents from the province of Granada (Spain). The instruments used were the Self-concept Questionnaire Form-5, Test for the Identification of Disorders in the Use of Alcohol-AUDIT, Test for the Nicotine-FTND Unit and an ad-hoc questionnaire to control the practice of physical activity. In this research, a model of structural equations was presented that was adjusted appropriately $(\mathrm{X} 2=79.476, \mathrm{gl}=8, \mathrm{p}=0,000, \mathrm{CFI}=0,968, \mathrm{NFI}=0,964, \mathrm{IFI}=0,968, \mathrm{RMSEA}=$ $0,065)$. The results obtained show a positive relationship between alcohol consumption and tobacco consumption, and negative relationships between family, emotional and academic self-concept with alcohol consumption.

KEYWORDS: self-concept; tobacco; alcohol; physical activity; teenagers.

\section{INTRODUCCIÓN}

La adolescencia es un periodo de transición entre la niñez y la adultez, produciéndose multitud de cambios a nivel físico, cognitivo y social, como el desarrollo de los caracteres sexuales o la adquisición del rol adulto (Booker y Dunsmore, 2017). La etapa adolescente supone un cambio radical en el estilo de vida de la persona, produciéndose cambios en las relaciones sociales y en su estilo de vida, culminando esto con el abandono del hogar familiar, la inserción en el mercado laboral o el inicio de estudios superiores (Jolliffe, Farrington, Piquero, MacLeod y van de Weijer, 2017). Además, los cambios sociales que se producen al abandonar la niñez y entrar en la etapa adulta adquiriendo la mayoría de edad legal, modifican la conducta de la persona al adquirir un rol diferente a nivel social (Bewick, Koutsopoulou, Miles, Slaa y Barkham, 2010; Ray, Frick, Thornton, Wall Myers, Steinberg y Cauffman, 2017). Durante la adquisición del rol adulto, se suelen manifestar conductas propias de la adultez y conductas más infantiles, debido a que el sujeto no habrá afianzado su identidad personal adulta de forma completa hasta completar la adolescencia (Haapanen y Tervo, 2012; Klemanski, Curtiss, McLaughlin y Nolen-Hoeksema, 2017). Motivado por esta inestabilidad emocional, en las sociedades posindustriales, los adolescentes suelen presentar un carácter inestable, siendo muy influenciables por su grupo de iguales, ya que no han afianzado los mecanismos necesarios para controlar los estresores propios de la adultez (Bewick et al., 2010; Rutland y Killen, 2017).

El consumo de tabaco y alcohol goza de gran popularidad entre la población adolescente, llegando a representar el paso de la niñez a la adultez, e incluso reafirmando la condición de adulto en el adolescente (Mezquita, Stewart, Kuntsche y Grant, 2016; Gaete y Araya, 2017). El consumo de estas sustancias 
provoca patrones de uso y dependencia que acarrearán futuras enfermedades crónicas y diversos problemas de salud asociados a su consumo (Kobiella et al., 2014). En la actualidad el consumo de drogas legales como el alcohol y el tabaco están descendiendo en la población adolescente, aunque los niveles siguen siendo altos, sobre todo en el caso del alcohol, debido a que las políticas sanitarias llevadas a cabo para la reducción del consumo de tabaco están teniendo efecto en la sociedad (Hernández, Orozco y Ríos, 2017). La adolescencia suele ser la etapa en la que se inicia el consumo de dichas sustancias, debido a la ausencia del control parental, la influencia y presión social que ejerce el grupo de iguales, además de los cambios contextuales y cognitivos que se producen (Carver, Elliott, Kennedy y Hanley, 2017). En la sociedad actual, el alcohol actúa como medio de socialización, debido a que su ingesta modifica las sensaciones y emociones, alterando los procesos de pensamiento y juicio crítico, provocando una fase de excitación que predispone a relacionarse con los iguales (Mezquita et al., 2016; Pegg, O'Donnell, Lala y Barber, 2017). El tabaco ha perdido gran parte de su atractivo social, aunque produce una fuerte adicción, debido al alto contenido en nicotina y demás sustancias que contiene, las cuales alteran el estado emocional del sujeto, generando una dependencia hacia la sustancia en cuestión (Kobiella et al., 2014).

El consumo de alcohol y tabaco está socialmente aceptado en la sociedad, debido a que son drogas blandas o legales, aunque conllevan unos riesgos y efectos perjudiciales para la salud de extrema gravedad. En el caso del alcohol, según la OMS (2015) el consumo de esta sustancia provoca más de tres millones de muertes al año en todo el mundo, además de constituir un factor de riesgo a la hora de padecer diversas enfermedades y trastornos cardiovasculares como la cirrosis o el cáncer hepático, entre otros (Maurage, Joassin, Speth, Modave, Philippot y Campanella, 2012; Connor, 2017; Eliott, Forster, McDonough, Bowd y Crabb, 2017). El tabaco provoca según la OMS (2015) más se de seis millones de muertes al año en todo el mundo, duplicando al alcohol, además el consumo de esta sustancia se relaciona de forma directa con el padecimiento de enfermedades como el enfisema pulmonar, la enfermedad pulmonar obstructiva crónica, diversas cardiopatías y el cáncer de pulmón (Haldorsen, Martinsen, Kjærheim y Grimsrud, 2017; Vrana, Kern y Anderson, 2017). Tras el análisis de estos datos se hace necesario controlar el consumo de ambas sustancias en la etapa adolescente, por representar el inicio del consumo y adicción a ellas, promoviendo su prevención y control por parte de los estamentos sanitarios estatales (Kuipers, Brandhof, Monshouwer, Stronks y Kunst, 2017).

Como alternativa al desarrollo de conductas dañinas durante la adolescencia, se plantea la necesidad de promover políticas sociales relacionadas con la adquisición de conductas saludables de práctica de actividad física, debido a los innumerables beneficios que conlleva, como la reducción del riesgo de padecer enfermedades cardiovasculares, mejoras a nivel cognitivo, fisiológico y social (Lewis, Napolitano, Buman, Williams y Nigg, 2017). En la adolescencia se produce un abandono de la práctica de actividad física (Bonet, Parrado y Capdevila, 2017), motivada por diversos factores culturales, aunados a la menor disponibilidad de tiempo para el ocio y el tiempo libre, por lo que debe ser tarea fundamental de los estados la adquisición de hábitos de práctica de actividad 
física por parte de la población, desde las edades más tempranas (Schlechter, Rosenkranz, Milliken y Dzewaltowski, 2017).

Durante la adolescencia se interrelacionan multitud de factores cognitivos y sociales que influyen sobremanera en el desarrollo personal, propiciando la adquisición de patrones que se repetirán durante la adultez, ya sean saludables, como la práctica de actividad física, o dañinos, como el consumo de sustancias nocivas; por estos motivos se hace imprescindible analizar los factores cognitivos que influyen en la adolescencia. Por lo que en la presente investigación se considera la necesidad de analizar el autoconcepto los adolescentes, entendido como una representación mental de cómo un individuo se ve a sí mismo (Hattie, 2014), compuesta por diversos factores -sociales, emocionales, físicos, familiares y académicos- (García y Musitu, 1999; Shavelson, Hubner y Stanton, 1976).

Multitud de investigaciones han analizado el autoconcepto en relación con diversas variables y en diferentes etapas, siendo la adolescencia una de las más estudiadas (Zurita, Castro, Álvaro, Rodríguez y Pérez, 2016). En su estudio, Moreno, Moreno y Cervelló, (2009) encontraron que los adolescentes no fumadores tenían una mejor imagen corporal; por otro lado, Dudovit, Li y Chung (2013) mostraron que el autoconcepto predice comportamientos relacionados con el consumo abusivo de alcohol en adolescentes, haciendo patente la necesidad de intervenir en el desarrollo de un autoconcepto saludable durante la niñez y la adolescencia para evitar problemas derivados de esto. Ante esta panorámica, resulta primordial continuar investigando sobre las relaciones entre factores cognitivos, sociales y contextuales que afecten al adolescente e influyan sobre la elección de hábitos saludables y/o dañinos (Jackson, Von Eye, Fitzgerald, Zhao y Witt, 2010; Rhodes, Devlin, Steinberg y Giovannetti, 2017).

Partiendo de la bibliografía analizada, se plantea un supuesto teórico que sustenta esta investigación mediante el siguiente modelo hipotético (Figura 1) con los siguientes factores; Factor 1: Autoconcepto Académico (AA), Factor 2: Autoconcepto Social (AS), Factor 3: Autoconcepto Familiar (AFM), Factor 4: Autoconcepto Físico (AF), Factor 5: Autoconcepto Emocional (AE), Factor 6: Práctica de Actividad Física (A. Física), Factor 7: Consumo de alcohol (ALCOHOL) y Factor 8: Consumo de tabaco (TABACO). 


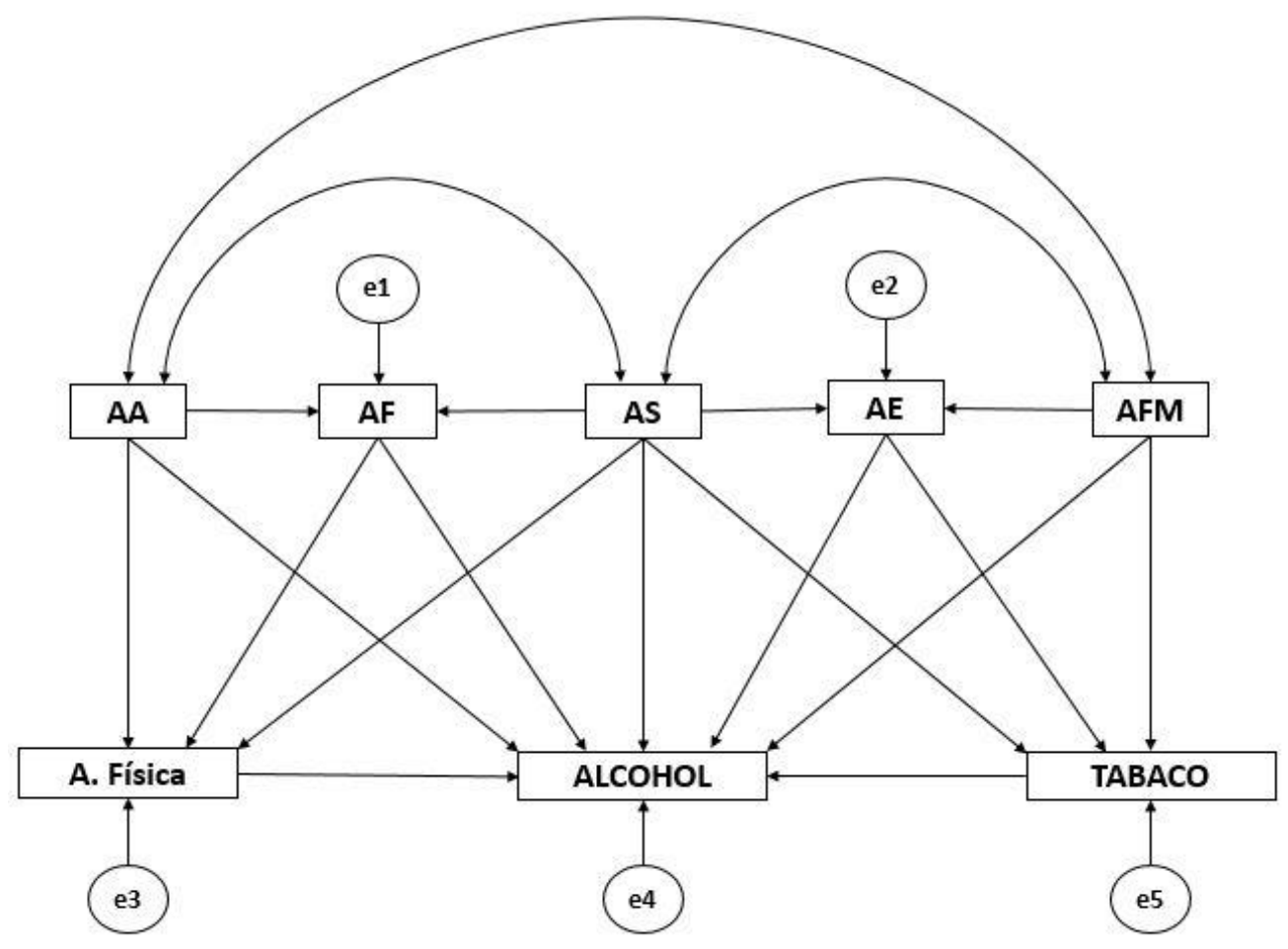

Figura 1. Modelo hipotético de consumo de sustancias nocivas, práctica de actividad física y autoconcepto.

Nota. A. AA: Autoconcepto Académico; AF: Autoconcepto Físico; AS: Autoconcepto Social; Física: AE: Autoconcepto Emocional; AFM: Autoconcepto Familiar; A. Física: Actividad Física; ALCOHOL: Consumo de alcohol; TABACO: Consumo de tabaco.

En el modelo desarrollado, el cual se basa en el planteado por Chacón, Castro, Caracuel, Padial, Collado y Zurita (2016) se pretende comprobar la relación entre todas las dimensiones del autoconcepto y el consumo de alcohol, debido a la existencia de multitud de estudios que analizan dicha relación en la adolescencia, indicando que el consumo de dicha sustancia se encuentra determinada por factores psicosociales (Murray, Farrington y Sekol, 2012; Mezquita et al., 2016). También se pretende comprobar la asociación del autoconcepto académico, físico y social con la práctica de actividad física. No se ha contemplado la relación con el autoconcepto emocional y familiar, debido a que en la adolescencia se produce un alejamiento por parte del sujeto del núcleo familiar (Bewick et al., 2010). Se ha buscado establecer las pertinentes relaciones con la finalidad de que no se vean afectados los índices de ajuste del modelo, y así obtener un modelo sintetizado y parsimonioso (Marsh, 2007).

El autoconcepto académico, el autoconcepto social y el autoconcepto familiar actúan en el modelo como variables exógenas, mientras que el autoconcepto físico y emocional, así como el consumo de alcohol y tabaco y la práctica de actividad física actúan como variables endógenas. Las flechas bidireccionales (covarianzas) relacionan las variables exógenas, mientras que las flechas unidireccionales revelan los efectos (directos e indirectos) entre las variables endógenas empleadas. Se asocian términos de error de predicción a las variables endógenas, las cuales reciben el efecto de otras y precisan de variables error. La estimación de los parámetros fue realizada a través del método de 
máxima verosimilitud (ML) por ser coherente, no sesgado e invariante al tipo de escala.

De este modo, este estudio plantea como objetivos: a) analizar y establecer las relaciones existentes entre las diferentes dimensiones del autoconcepto, las variables asociadas al consumo de sustancias dañinas (alcohol y tabaco) y la práctica de actividad física; b) definir y contrastar un modelo explicativo de consumo de sustancias nocivas y práctica de actividad física en función del autoconcepto y sus dimensiones en adolescentes españoles; c) analizar el efecto del autoconcepto en el consumo de alcohol y tabaco y en la práctica de actividad física a partir del modelo explicativo confeccionado.

\section{MÉTODO}

\section{Participantes}

Se realizó un estudio descriptivo, exploratorio y de corte transversal en el que participaron un total de 2.134 adolescentes españoles, de los cuales 1.062 eran chicos $(49,8 \%)$ y 1.072 eran chicas $(50,2 \%)$, con edades comprendidas entre los 15 y los 18 años $(M=15,93$ años; $D T=0,853)$, provenientes de centros de Educación Secundaria Obligatoria (ESO) de la provincia de Granada. De un total de 18.930 estudiantes matriculados para el curso académico 2.014/2.015 (datos provistos por la Consejería de Educación), se estableció una muestra representativa de 2.134 estudiantes de Educación Secundaria Obligatoria (ESO) (error muestral de 0,$02 ; I C=95,5 \%$ ) usando técnicas de muestreo aleatorio estratificado, como indican Santos, Muñoz, Juez y Cortiñas (2003). Los centros educativos y estudiantes colaboraron voluntariamente en el desarrollo de la investigación.

\section{Variables e Instrumentos}

La presente investigación tomó como referencia las siguientes variables e instrumentos de medida:

Cuestionario Autoconcepto Forma-5 (AF-5). Este instrumento fue elaborado por García y Musitu (1999) y se fundamenta en el modelo teórico de Shavelson et al. (1976). Se compone de 30 ítems que se puntúan mediante una escala tipo Likert de 5 opciones, donde 1 es "Nunca" y 5 es "Siempre". El autoconcepto queda agrupado en cinco dimensiones según este instrumento, las cuales son: autoconcepto académico (ítems 1, 6, 11, 16, 21 y 26), autoconcepto social (ítems 2, 7, 12, 17, 22 y 27), autoconcepto emocional (ítems 3, 8, 13, 18, 23 y 28), autoconcepto familiar (ítems 4, 9, 14, 19, 24 y 29) y autoconcepto físico (ítems $5,10,15,20,25$ y 30). En el estudio de García y Musitu (1999) se estableció una fiabilidad (determinada mediante el coeficiente alpha de Cronbach) de $\alpha=0,810$, valor similar al detectado en este trabajo de investigación $(\alpha=0,833)$.

Escala de consumo de tabaco, extraída del cuestionario "Fagerström Test for Nicotine Dependence (FTND)", el cual fue desarrollado por Heatherton, Kozlowski, Frecker y Fagerström (1991) y traducido al castellano por Villareal- 
González (2009). Este instrumento permite medir la cantidad de cigarros que fuma el adolescente, su impulso por fumar y la dependencia que tiene hacia la nicotina. Se compone de 6 ítems, cuatro de los cuales son de tipo dicotómico $(0=$ No y $1=\mathrm{Si})$, mientras que los dos restantes se evalúan mediante una escala tipo Likert de tres opciones. La fiabilidad para este instrumento en este estudio fue de $\alpha=0,956$, valor prácticamente idéntico al obtenido por Villareal-González (2009) en el trabajo original $(\alpha=0,970)$.

Escala de consumo de alcohol, extraída del instrumento "Alcohol Use Disorders Identification Test (AUDIT)". Este fue desarrollado por Saunders et al. (1993) y traducido al castellano por Rubio (1998). Se compone de 10 ítems, siendo los ocho primeros valorados mediante una escala de tipo Likert de cinco opciones, donde 0 es "Nunca" y 5 "A Diario". Las dos últimas cuestiones se cuantifican mediante una escala de tipo Likert de tres opciones $(0,2$ y 4$)$. El instrumento es valorado mediante sumatoria que oscila entre 0 y 40 puntos y concreta dimensiones por factores. En el estudio original Rubio (1998) obtuvo una fiabilidad de $\alpha=0,800$, mientras que en la presente investigación fue de $\alpha=$ 0,832 .

Cuestionario Ad-hoc para medir la práctica de actividad física, en el que se preguntada si practicaban o no actividad física, con respuesta dicotómica $(0=\mathrm{No}$; $1=\mathrm{Si})$.

\section{Procedimiento}

En primer lugar se procedió a solicitar la colaboración de los participantes a través de una carta informativa elaborada desde el Área de Corporal de la Universidad de Granada. En esta se detallaba la naturaleza y objetivos del estudio a realizar, así como se solicitaba el consentimiento de los participantes y sus tutores legales. Posteriormente se realizó la recogida de datos. Un total 2.211 estudiantes de Educación Secundaria Obligatoria (ESO) tomaron parte en el estudio, teniendo que invalidar 77 cuestionarios por encontrarse mal cumplimentados; configurando una muestra final de 2.134 participantes. La aplicación de los instrumentos se realizó en horario lectivo en el centro descrito sin que ocurriese ningún tipo de incidencia. Asimismo, los investigadores estuvieron presentes con el fin de asegurar una correcta aplicación de los instrumentos. Se aseguró el anonimato a todos los estudiantes, los cuales participaron de manera voluntaria y respetando el acuerdo sobre ética de Investigación de Helsinki. El Comité Ético de Investigación de la Universidad de Granada aprobó este estudio.

\section{Análisis de los Datos}

Se utilizó el software estadístico IBM SPSS® en su versión 24 para Windows con el fin de realizar los análisis descriptivos básicos (medias y frecuencias). Se empleó el programa IBM AMOS ${ }^{2} 23$ para analizar las relaciones y efectos existentes entre los constructos implicados del modelo estructural diseñado. Para ello se concretó un modelo de análisis de rutas con las siguientes variables observables: autoconcepto académico (AA), autoconcepto físico (AF), 
autoconcepto social (AS), autoconcepto emocional (AE), autoconcepto familiar (AFA), práctica de actividad física (A. Física), consumo de alcohol (Alcohol) y consumo de tabaco (Tabaco). El ajuste del modelo fue comprobado con el fin de verificar la compatibilidad del mismo y la información empírica obtenida. La fiabilidad del ajuste fue realizada en base a los criterios de bondad de ajuste (Marsh, 2007, p.785). En el caso del Chi-cuadrado, los valores no significativos asociados a $p$ indican un buen ajuste del modelo. El valor del índice de ajuste comparativo (CFI) será aceptable con valores superiores a 0,90 y excelente para valores superiores a 0,95. El índice de ajuste normalizado (NFI) deberá ser superior a 0,90. El valor del índice de incremento de ajuste (IFI) será aceptable con valores superiores a 0,90 y excelente para valores superiores a 0,95 . Por último, el valor del error cuadrático medio de aproximación (RMSEA) será excelente si es inferior a 0,05 y aceptable si es inferior a 0,08.

\section{RESULTADOS}

Los valores obtenidos en la evaluación de ajuste del modelo indican un buen ajuste en todos los índices. El Chi-cuadrado presentó un valor significativo de $p$ ( $\mathrm{X} 2=79,476 ; g l=8 ; p=0,000)$, aunque debe considerarse que este estadístico, como índice, no tiene límite superior. Asimismo, no puede interpretarse de manera estandarizada, además del problema que plantea su sensibilidad al tamaño muestral. De este modo, se emplean otros índices de ajuste estandarizados que son menos sensibles al tamaño muestral. El índice de ajuste comparativo (CFI) mostró un valor de 0,968, el cual es excelente. El índice de ajuste normalizado (NFI) y el índice de incremento de ajuste (IFI) muestran valores de 0,964 y 0,968 respectivamente, los cuales son también excelentes. Finalmente, el error cuadrático medio de aproximación (RMSEA) obtiene un valor aceptable de 0,065.

La Figura 2 revela los valores estimados de los parámetros. Su magnitud ha de ser adecuada y los efectos significativamente distintos de cero. Tampoco deben obtenerse estimaciones impropias como varianzas negativas. 


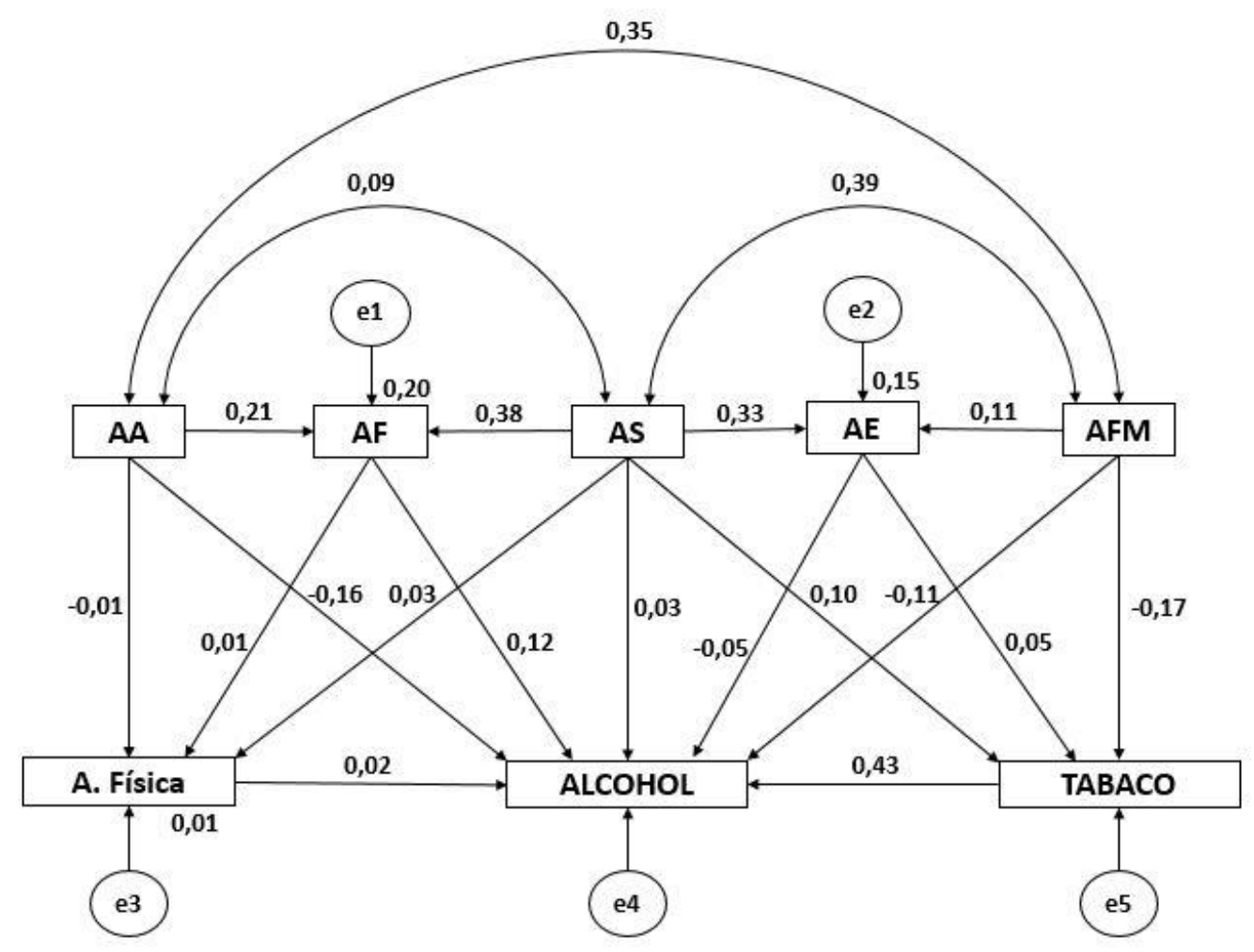

Figura 2. Modelo de ecuaciones estructurales.

Nota. A. AA: Autoconcepto Académico; AF: Autoconcepto Físico; AS: Autoconcepto Social; Física: AE: Autoconcepto Emocional; AFM: Autoconcepto Familiar; A. Física: Actividad Física; ALCOHOL: Consumo de alcohol; TABACO: Consumo de tabaco.

La Tabla 1 muestra relaciones estadísticamente significativas al nivel de 0,001 en la mayoría de relaciones entre variables excepto en siete. Los pesos con mayor índice de regresión se corresponden a la relación entre consumo de tabaco y alcohol, seguido de las relaciones entre autoconcepto social y físico, autoconcepto social y emocional, autoconcepto académico y físico, los cuales muestran una relación positiva y directa. Asimismo, se observan relaciones negativas entre el autoconcepto familiar y el consumo de tabaco, y entre el autoconcepto académico y el consumo de alcohol. También existe una relación negativa entre el autoconcepto familiar y el consumo de alcohol, y entre el autoconcepto emocional y el consumo de dicha sustancia. Atendiendo al modelo planteado, para la presente muestra poblacional, la práctica de actividad física no se relaciona con el resto de variables analizadas en este estudio. En el caso de las relaciones que no son estadísticamente significativas, el ratio crítico (C.R.) es inferior a 2, ya que los valores superiores a 2 en este coeficiente suponen que el parámetro es diferente de 0 de forma estadísticamente significativa a nivel de 0,001 (Byrne, 2013). Por tanto, se afirma que las escalas empleadas en las variables mencionas no poseen validez convergente. 
Tabla 1. Pesos de regresión y pesos estandarizados de regresión

\begin{tabular}{|c|c|c|c|c|c|c|c|}
\hline \multirow{2}{*}{\multicolumn{3}{|c|}{ Relaciones entre variables }} & \multirow{3}{*}{$\begin{array}{r}\text { Estimaciones } \\
0,218\end{array}$} & \multirow{3}{*}{$\begin{array}{r}\text { S.E. } \\
0,021\end{array}$} & \multirow{3}{*}{\multicolumn{2}{|c|}{$\begin{array}{r}\mathbf{P . R} . \\
\mathbf{P} \\
* \star \star\end{array}$}} & \multirow{3}{*}{$\begin{array}{r}\text { P.E.R. } \\
\text { Estimaciones } \\
0,206 \\
\end{array}$} \\
\hline & & & & & & & \\
\hline AF & $<---$ & AA & & & & & \\
\hline AF & $\leftarrow-$ & AS & 0,422 & 0,022 & 19,289 & $* * *$ & 0,376 \\
\hline$A E$ & $<---$ & AS & 0,361 & 0,024 & 15,321 & $* * *$ & 0,331 \\
\hline$A E$ & $<---$ & AFM & 0,110 & 0,021 & 5,310 & *** & 0,115 \\
\hline A.Física & $<---$ & AA & $-0,009$ & 0,014 & $-0,662$ & 0,508 & $-0,015$ \\
\hline A.Física & $<---$ & AF & 0,007 & 0,015 & 0,482 & 0,630 & 0,012 \\
\hline Tabaco & $<---$ & AFM & $-0,612$ & 0,082 & $-7,422$ & $* * *$ & $-0,173$ \\
\hline Tabaco & $<---$ & $\mathrm{AE}$ & 0,169 & 0,085 & 1,985 & 0,047 & 0,046 \\
\hline Tabaco & $<--$ & AS & 0,390 & 0,098 & 3,984 & $* * *$ & 0,097 \\
\hline A.Física & $<---$ & AS & 0,022 & 0,016 & 1,399 & 0,162 & 0,033 \\
\hline Alcohol & $<---$ & AS & 0,240 & 0,176 & 1,367 & 0,172 & 0,031 \\
\hline Alcohol & $<---$ & A.Física & 0,240 & 0,213 & 1,130 & 0,258 & 0,021 \\
\hline Alcohol & $<---$ & Tabaco & 0,825 & 0,036 & 22,695 & $* * *$ & 0,430 \\
\hline Alcohol & $<---$ & AF & 0,818 & 0,143 & 5,735 & $* * *$ & 0,120 \\
\hline Alcohol & $<---$ & $\mathrm{AE}$ & $-0,377$ & 0,143 & $-2,631$ & 0,009 & $-0,053$ \\
\hline Alcohol & $<---$ & AA & $-1,149$ & 0,148 & $-7,779$ & $* * *$ & $-0,159$ \\
\hline Alcohol & $<---$ & AFM & $-0,714$ & 0,149 & $-4,800$ & $* * *$ & $-0,105$ \\
\hline
\end{tabular}

${ }^{1}$ P.R., Pesos de Regresión; P.E.R., Pesos Estandarizados de Regresión; S.E., Estimación del error; C.R., Ratio Crítico.

${ }^{2}$ A. Física: Actividad Física; AA: Autoconcepto Académico; AS: Autoconcepto Social; AFM: Autoconcepto Familiar; AF: Autoconcepto Físico; AE: Autoconcepto Emocional.

$3^{* \star \star}$ Relación entre variables estadísticamente significativa al nivel 0,005.

\section{DISCUSIÓN}

Esta investigación analiza el modelo de autoconcepto compuesto por cinco dimensiones -académica, física, social, emocional y familiar- (Fuentes, García, Gracia y Lila, 2011) en adolescentes, así como su relación con la práctica de actividad física, el consumo de alcohol y tabaco. El modelo de ecuaciones estructurales que se ha propuesto se ajusta de forma adecuada, fundamentando los resultados obtenidos en investigaciones previas realizadas en contextos similares (Bustos, Oliver y Galiana, 2015; Dudovitz, Li y Chung, 2013; Jackson et al., 2010; McKay, Sumnall, Cole y Percy, 2012; Zurita et al., 2016).

Como principales resultados se indica que todas las dimensiones del autoconcepto se relacionan entre ellas, excepto la dimensión emocional y familiar. Sin embargo, estudios como los de Bustos et al. (2015) y RodríguezFernández, Droguett y Revuelta (2012) obtuvieron una relación directa entre la dimensión emocional y la familiar. Es necesario ser cauteloso con estos resultados, ya que esta ausencia de relación puede deberse a la disminución de las relaciones familiares que se produce durante la adolescencia, por entrar en una etapa de mayor independencia social y emocional (Haapanen y Tervo, 2012; Berg, Kiviruusu, Karvonen, Rahkonen y Huurre, 2017).

El consumo de alcohol se relaciona de forma directa con el consumo de tabaco, mostrando una relación directa de gran fortaleza, debido a que el consumo de ambas sustancias se suele simultanear. Multitud de investigaciones muestran 
como los jóvenes que consumen una mayor cantidad de alcohol son los que más fuman, debido a que ambas drogas pueden consumirse a la vez, potenciando una los efectos de la otra (Lindgren, Neighbors, Gasser, Ramirez y Cvencek, 2017; Matuszka, Bácskai, Czobor y Gerevich, 2017). Al analizar el consumo de tabaco en relación con el autoconcepto familiar se ha encontrado una relación negativa, mostrando como la familia influye sobremanera en el consumo de sustancias nocivas. Chartier, Thomas y Kendler (2017) indican que el consumo de alcohol y tabaco puede relacionarse con conductas de evitación o escape de situaciones problemáticas familiares, lo que explicaría los resultados que indican que los adolescentes que poseen un autoconcepto familiar pobre son los que más tabaco consumen (Murray, Durazzo, Mon, Schmidt y Meyerhoff, 2015).

Cuando se analizó la asociación entre consumo de alcohol y autoconcepto, se encuentran relaciones positivas con las dimensiones física y social, mientras que se observa una relación negativa con la académica. Esto puede deberse a que en la adolescencia imperan los motivos sociales para el consumo de alcohol, lo que justificaría la relación entre el consumo de dicha sustancia y la dimensión social del autoconcepto (Pegg et al., 2017). También se asocia el consumo de sustancias nocivas a un rendimiento académico bajo y al abandono escolar en las etapas pre-puberal y adolescente (Kelly, Chan, Mason y Williams, 2015). La ingesta de drogas, legales o ilegales, se asocia a situaciones familiares disfuncionales, en las que la influencia del entorno familiar es escasa y el sujeto se apoya en el grupo de iguales fundamentalmente, careciendo de disciplina (Maurage et al., 2012).

Al analizar la práctica de actividad física en relación con el resto de variables no se ha encontrado asociación, aunque en estudios como el de Moreno, Moreno y Cervelló (2013) se encontró una relación entre el autoconcepto físico y la intención de ser físicamente activo, al igual que en el estudio de Revuelta, Esnaola y Goñi (2016).

Es necesario indicar las principales limitaciones con las que cuenta esta investigación, entre las que cabría destacar el uso de un diseño descriptivo de corte transversal, que no permite establecer relaciones causa-efecto entre las variables. Además hubiese sido interesante ampliar el número de variables analizadas en relación con la práctica de actividad física en la adolescencia, así como incorporar un mayor número de variables psicológicas, como los niveles de autoestima y ansiedad.

Como principales conclusiones de la investigación, se extrae que el modelo de ecuaciones estructurales planteado se ajusta con una buena fiabilidad y de forma apropiada, por lo que genera una visión amplia de la relación entre los factores psicológicos como el autoconcepto en relación con el consumo de sustancias nocivas. Se han encontrado relaciones positivas entre las dimensiones social y física del autoconcepto con el consumo de alcohol, así como una relación negativa entre la dimensión académica y el consumo de dicha sustancia. Del mismo modo, el consumo de tabaco se relaciona de forma directa con el consumo de alcohol, actuando el autoconcepto como un factor de riesgo en el consumo de sustancias nocivas en adolescentes. No se ha encontrado relación 
entre la práctica de actividad física y el resto de variables analizadas en el modelo propuesto.

\section{Referencias Bibliográficas}

Berg, N., Kiviruusu, O., Karvonen, S., Rahkonen, O., y Huurre, T. (2017). Pathways from poor family relationships in adolescence to economic adversity in mid-adulthood. Advances in Life Course Research, 32, 65-78. https://doi.org/10.1016/j.alcr.2016.07.001

Bewick, B., Koutsopoulou, G., Miles, J., Slaa, E. y Barkham, M. (2010). Changes in undergraduate students' psychological well-being as they progress through university. Studies in Higher Education, 35, 633-645. https://doi.org/10.1080/03075070903216643

Bonet, J.; Parrado, E. y Capdevila, L. (2017). Efectos agudos del ejercicio físico sobre el estado de ánimo y la HRV / Acute Effects of Exercise on Mood And HRV. Revista Internacional de Medicina y Ciencias de la Actividad Física y el Deporte, 17(65), 85-100. http://dx.doi.org/10.15366/rimcafd2017.65.006

Booker, J. A., y Dunsmore, J. C. (2017). Affective Social Competence in Adolescence: Current Findings and Future Directions. Social Development, 26(1), 3-20. https://doi.org/10.1111/sode.12193

Bustos, V., Oliver, A. y Galiana, L. (2015). Validación del Autoconcepto Forma 5 en Universitarios Peruanos: Una herramienta para la psicología positiva: Psicología Reflexao e Critica, 28, 690-697. https://doi.org/10.1590/16787153.201528406

Byrne, B. M. (2013). Structural equation modelling with AMOS: Basic concepts, applications, and programming. Routledge: New York. https://doi.org/10.4324/9780203726532

Carver, H., Elliott, L., Kennedy, C., y Hanley, J. (2017). Parent-child connectedness and communication in relation to alcohol, tobacco and drug use in adolescence: An integrative review of the literature. Drugs: Education, Prevention and Policy, 24(2), 119-133. https://doi.org/10.1080/09687637.2016.1221060

Chacón, R., Castro, M., Caracuel, R., Padial, R., Collado, D. y Zurita, F. (2016). Perfiles de consumo de alcohol y tabaco en adolescentes andaluces de primer ciclo de educación secundaria. Health and Addictions Journal, 16, 93-104. https://doi.org/10.21134/haaj.v16i2.266

Chartier, K. G., Thomas, N. S., y Kendler, K. S. (2017). Interrelationship between family history of alcoholism and generational status in the prediction of alcohol dependence in US Hispanics. Psychological medicine, 47(1), 137147. https://doi.org/10.1017/S0033291716002105

Connor, J. (2017). Alcohol consumption as a cause of cancer. Addiction, 112(2), 222-228. https://doi.org/10.1111/add.13477

Dudovitz, R. N., Li, N. y Chung, P. J. (2013). Behavioral self-concept as predictor of teen drinking behaviors. Academic Pediatrics, 13, 316-321. https://doi.org/10.1016/j.acap.2013.03.005

Eliott, J., Forster, A. J., McDonough, J., Bowd, K., y Crabb, S. (2017). An examination of Australian newspaper coverage of the link between alcohol 
and cancer 2005 to 2013. BMC Public Health, 18(1), 47-61. https://doi.org/10.1186/s12889-017-4569-0

Fuentes, M., García, J. F., Gracia, E. y Lila, M. (2011). Autoconcepto y ajuste psicosocial en la adolescencia. Psicothema, 23, 7-12. https://doi.org/10.20882/adicciones.148

Gaete, J., y Araya, R. (2017). Individual and contextual factors associated with tobacco, alcohol, and cannabis use among Chilean adolescents: A multilevel study. Journal of adolescence, 56, 166-178. https://doi.org/10.1016/i.adolescence.2017.02.011

García, F. y Musitu, G. (1999). AF5: Autoconcepto Forma 5. Madrid: TEA Ediciones.

Haapanen, M. y Tervo, H. (2012). Migration of the highly educated: Evidence from residence spell of university graduates. Journal of Regional Science, 52, 587-605. https://doi.org/10.1111/j.1467-9787.2011.00745.x

Haldorsen, T., Martinsen, J. I., Kjærheim, K., y Grimsrud, T. K. (2017). Adjustment for tobacco smoking and alcohol consumption by simultaneous analysis of several types of cancer. Cancer Causes $y$ Control, 28(2), 155-165. https://doi.org/10.1007/s10552-016-0847-x

Hattie, J. (2014). Self-concept. Psychology Press: New York. https://doi.org/10.4324/9781315802183

Heatherton, T., Kozlowski, L., Frecker, R. y Fagerström, K. O. (1991). The Fagerström Test of Nicotine Dependence: a revision of the Fagerström Tolerance Questionnaire. British Journal of Addiction, 86, 1119-1127. https://doi.org/10.1111/j.1360-0443.1991.tb01879.x

Hernández, E. M., Orozco, I. C., y Ríos, J. D. (2017). Estado del Arte sobre el diseño, análisis y evaluación de Políticas de Reducción de Daño por Consumo de Sustancias Psicoactivas, en Europa y América entre 2003 a 2013. Health and Addictions/Salud y Drogas, 17(2), 5-16. https://doi.org/10.21134/haaj.v17i2.275

Jackson, L., Von Eye, A., Fitzgerald, H., Zhao, Y. y Witt, E. A. (2010). Selfconcept, self-esteem, gender, race and information technology use. Computers in Human Behavior, 26, 323-328. https://doi.org/10.1016/j.chb.2009.11.001

Jolliffe, D., Farrington, D. P., Piquero, A. R., MacLeod, J. F., y van de Weijer, S. (2017). Prevalence of life-course-persistent, adolescence-limited, and late-onset offenders: A systematic review of prospective longitudinal studies. Aggression and violent behavior, 33, 4-14. https://doi.org/10.1016/j.avb.2017.01.002

Kelly, A., Chan, G., Mason, W. y Williams, J. (2015). The relationship between psychological distress and adolescent polydrug use. Psychology of Addictive Behaviors, 29, 787-793. https://doi.org/10.1037/adb0000068

Klemanski, D. H., Curtiss, J., McLaughlin, K. A., y Nolen-Hoeksema, S. (2017). Emotion Regulation and the Transdiagnostic Role of Repetitive Negative Thinking in Adolescents with Social Anxiety and Depression. Cognitive therapy and research, 41(2), 206-219. https://doi.org/10.1007/s10608016-9817-6

Kobiella, A., Ripke, S., Kroemer, N. B., Vollmert, C., Vollstad- Klein, S., Ulshofer, D. y Smolka, M. N. (2014). Acute and chronic nicotine effects on behaviour 
and brain activation during intertemporal decission making. Addiction Biology, 19, 918-930. https://doi.org/10.1111/adb.12057

Kuipers, M. A., Brandhof, S. D., Monshouwer, K., Stronks, K., y Kunst, A. E. (2017). Impact of laws restricting the sale of tobacco to minors on adolescent smoking and perceived obtainability of cigarettes: an intervention-control pre-post study of 19 European Union countries. Addiction, 112(2), 320-329. https://doi.org/10.1111/add.13605

Lewis, B. A., Napolitano, M. A., Buman, M. P., Williams, D. M., y Nigg, C. R. (2017). Future directions in physical activity intervention research: expanding our focus to sedentary behaviors, technology, and dissemination. Journal of behavioral medicine, 40(1), 112-126. https://doi.org/10.1007/s10865-016-9797-8

Lindgren, K. P., Neighbors, C., Gasser, M. L., Ramirez, J. J., y Cvencek, D. (2017). A review of implicit and explicit substance self-concept as a predictor of alcohol and tobacco use and misuse. The American journal of drug and alcohol abuse, 43(3), 237-246. https://doi.org/10.1080/00952990.2016.1229324

Marsh, H. W. (2007). Handbook of Sport Psychology. Third Edition. Tenenbaum and R. C. Eklund: New Jersey.

Matuszka, B., Bácskai, E., Czobor, P., y Gerevich, J. (2017). Physical Aggression and Concurrent Alcohol and Tobacco Use Among Adolescents. International Journal of Mental Health and Addiction, 15(1), 90-99. https://doi.org/10.1007/s11469-015-9630-6

Maurage, P., Joassin, F., Speth, A., Modave, J., Philippot, P. y Campanella, S. (2012). Cerebral effects of binge drinking: Respective influences of global alcohol intake and consumption pattern. Clinical Neurophysiology, 123, 892-901. https://doi.org/10.1016/j.clinph.2011.09.018

McKay, M. T., Sumnall, H. R., Cole, J. C. y Percy, A. (2012). Self-esteem and self-efficacy: Associations with alcohol consumption in a sample of adolescents in Northern Ireland. Drugs: education, prevention and policy, 19, 72-80. https://doi.org/10.3109/09687637.2011.579585

Mezquita, L., Stewart, S., Kuntshe, E. y Grant, V. (2016). Estudio transcultural del modelo de cinco factores de motivos de consumo de alcohol en universitarios españoles y canadienses. Adicciones, 28, 215-220. https://doi.org/10.20882/adicciones.822

Moreno, J. A., Moreno, R. y Cervelló, E. (2009). Relación del autoconcepto físico con las conductas de consumo de alcohol y tabaco en adolescentes. Adicciones, 21, 147-154. https://doi.org/10.20882/adicciones.242

Moreno, J. A., Moreno, R., y Cervelló, E. (2013). El autoconcepto físico como predictor de la intención de ser físicamente activo. Psicología y salud, 17(2), 261-267.

Murray, D. E., Durrazo, T. C., Mon, A., Schmidt, T. P. y Meyerhoff, D. J. (2015). Brain perfusion in polysubstance users: Relationship to substance and tobacco use, cognition, and self-regulation. Drug and Alcohol Dependence, $150, \quad 120-128$. https://doi.org/10.1016/i.drugalcdep.2015.02.022

Murray, J., Farrington, D. y Sekol, I. (2012). Children's antisocial behaviour, mental health, drug use, and educational performance after parental 
incarceration: A systematic review and meta-analysis. Psychological Bulletin, 138, 175-210. https://doi.org/10.1037/a0026407

OMS (2015). Alcohol. Nota descriptiva № 349. Consultado en la Word Wide Web el 12 de Junio 2016: http://www.who.int/mediacentre/factsheets/fs349/es.

Pegg, K. J., O'Donnell, A. W., Lala, G., y Barber, B. L. (2017). The role of online social identity in the relationship between alcohol-related content on social networking sites and adolescent alcohol use. Cyberpsychology, Behavior, and Social Networking, 6, 1-6. https://doi.org/10.1089/cyber.2016.0665

Ramos, P.; Jiménez-Iglesias, A.; Rivera, F. y Moreno, C. (2016) Evolución de la práctica de la actividad física en los adolescentes españoles / Physical Activity Trends in Spanish Adolescents. Revista Internacional de Medicina y Ciencias de la Actividad Física y el Deporte, 16(62) pp.335-353. https://doi.org/10.15366/rimcafd2016.62.010

Ray, J. V., Frick, P. J., Thornton, L. C., Wall Myers, T. D., Steinberg, L., y Cauffman, E. (2017). Callous-unemotional traits predict self-reported offending in adolescent boys: The mediating role of delinquent peers and the moderating role of parenting practices. Developmental psychology, 53(2), 319-328. https://doi.org/10.1037/dev0000210

Revuelta, L., Esnaola, I., y Goñi, A. (2016). Relaciones entre el autoconcepto físico y la actividad físico-deportiva adolescente. Revista Internacional de Medicina y Ciencias de la Actividad Física y del Deporte, 16(63), 561-581, https://doi.org/10.15366/rimcafd2016.63.010

Rhodes, E., Devlin, K. N., Steinberg, L., y Giovannetti, T. (2017). Grit in adolescence is protective of late-life cognition: non-cognitive factors and cognitive reserve. Aging, Neuropsychology, and Cognition, 24(3), 321332. https://doi.org/10.1080/13825585.2016.1210079

Rodríguez-Fernández, A., Droguett, L. y Revuelta, L. (2012). School and Personal Adjustment in Adolescence: The Role of Academic Self-Concept and Perceived Social Support. Revista de Psicodidáctica, 17, 397-414. https://doi.org/10.1387/RevPsicodidact.3002

Rubio, G. (1998). Validación de la prueba para la identificación de trastornos por el uso de alcohol (AUDIT) en Atención Primaria. Revista Clínica Especializada, 198, 11-14.

Rutland, A., y Killen, M. (2017). Fair resource allocation among children and adolescents: The role of group and developmental processes. Child Development Perspectives, 11(1), 56-62. https://doi.org/10.1111/cdep.12211

Santos, J., Muñoz, P. Juez, J. y Cortiñas, A. (2003). Diseño de Encuestas para estudios de mercado. Técnicas de muestreo y análisis multivariante. Madrid: Editorial Ramón Areces.

Saunders, J., Aasland, O., Babor, T., De la Fuente, J. y Grant, M. (1993). Development of the Alcohol Use Disorders Identification Test (AUDIT): Who collaborative Project on early detection of persons with harmful alcohol consumption-II. Addiction, 88, 791-804. https://doi.org/10.1111/j.1360-0443.1993.tb00822.x

Schlechter, C. R., Rosenkranz, R. R., Milliken, G. A., y Dzewaltowski, D. A. (2017). Physical activity levels during youth sport practice: does coach 
training or experience have an influence?. Journal of sports sciences, 35(1), 22-28. https://doi.org/10.1080/02640414.2016.1154593

Shavelson, J., Hubner, J. J. y Stanton, G. C. (1976). Self-concept: validation of construct interpretations. Review of Educational Research, 46, 407-442. https://doi.org/10.3102/00346543046003407

Villareal-González, M. E. (2009). Un modelo estructural del consumo de drogas y conducta violenta en adolescentes escolarizados. Tesis Doctoral: Universidad Autónoma de Nuevo León (México).

Vrana, C. J., Kern, T., y Anderson, R. (2017). Assessment of Tobacco Use in Cancer Survivorship Research Among National Cancer Institutedesignated Cancer Centers or Affiliated Universities: A Systematic Review. Journal of Student Research, 6(1), 8-13.

Zurita, F., Castro, M., Álvaro, J. I., Rodríguez, S. y Pérez, A. J. (2016). Autoconcepto, Actividad física y Familia: Análisis de un modelo de ecuaciones estructurales. Revista de Psicología del Deporte, 25, 97-104.

Número de citas totales / Total references: 53 (100\%)

Numero de citas propias de la revista / Journal's own references: 2 $(3.77 \%)$

Rev.int.med.cienc.act.fís.deporte - vol. 19 - número 75 - ISSN: 1577-0354 